\title{
Construction of bivariate asymmetric copulas
}

\author{
Saikat Mukherjee ${ }^{a}$, Youngsaeng Lee ${ }^{1, b}$, Jong-Min Kim $^{c}$, Jun Jang ${ }^{d}$, Jeong-Soo Park ${ }^{b}$ \\ ${ }^{a}$ Department of Mathematics, National Institute of Technology, India; \\ ${ }^{b}$ Department of Statistics, Chonnam National University, Korea; \\ ${ }^{c}$ Division of Science and Mathematics, University of Minnesota-Morris, USA; \\ ${ }^{d}$ Center for Information Analysis, Chungnam National University, Korea
}

\begin{abstract}
Copulas are a tool for constructing multivariate distributions and formalizing the dependence structure between random variables. From copula literature review, there are a few asymmetric copulas available so far while data collected from the real world often exhibit asymmetric nature. This necessitates developing asymmetric copulas. In this study, we discuss a method to construct a new class of bivariate asymmetric copulas based on products of symmetric (sometimes asymmetric) copulas with powered arguments in order to determine if the proposed construction can offer an added value for modeling asymmetric bivariate data. With these newly constructed copulas, we investigate dependence properties and measure of association between random variables. In addition, the test of symmetry of data and the estimation of hyper-parameters by the maximum likelihood method are discussed. With two real example such as car rental data and economic indicators data, we perform the goodness-of-fit test of our proposed asymmetric copulas. For these data, some of the proposed models turned out to be successful whereas the existing copulas were mostly unsuccessful. The method of presented here can be useful in fields such as finance, climate and social science.
\end{abstract}

Keywords: Cramér-von Mises statistics, empirical copula, Fourier copula, maximum pseudolikelihood estimation, parametric bootstrap, pseudo-observations

\section{Introduction}

Copulas offer a useful tool in modeling the dependence among random variables. For example, Busababodhin and Amphanthong (2016) applied copula in the multivariate statistical process control and Kim (2014) used copula-GARCH for the modeling of dependence structure of Korea financial markets. In the literature, most of the existing copulas, however, are symmetric while data collected from the real world may exhibit asymmetric nature. This necessitates developing asymmetric copulas. Many researchers proposed some methods to construct asymmetric copulas; Rodríguez-Lallena and Úbeda-Flores (2004) introduced a class of bivariate copulas that generalizes some known families. Kim et al. (2011) and Mesiar and Najjari (2014) extended the method of Rodríguez-Lallena and Úbeda-Flores (2004) to construct new families of symmetric and asymmetric copulas. Alfonsi and Brigo (2005) described a new construction method for asymmetric copulas based on periodic functions. Liebscher (2008) introduced two methods to construct asymmetric multivariate copulas, which is close to what Khoudraji (1995) proposed earlier (Quessy and Kortbi, 2016). The first is connected

\footnotetext{
${ }^{1}$ Corresponding author: Department of Statistics, Chonnam National University, 77 Yongbong-ro, Buk-gu, Gwangju 61186, Korea. E-mail: yslee82@ejnu.net
}

Published 31 March 2018 / journal homepage: http://csam.or.kr

(c) 2018 The Korean Statistical Society, and Korean International Statistical Society. All rights reserved. 
with products of copulas while the second one is a generalization of the Archimedean copulas family (Di Bernardino and Rullière, 2015). Durante (2009) suggested a method to construct asymmetric copulas based on products of copulas with powered arguments. Wu (2014) proposed a new method of constructing asymmetric copulas using a mixture of basic copulas and a convex combination of asymmetric copulas that can exhibit different tail dependence along different directions. Di Bernardino and Rullière (2015) constructed multivariate family of copulas by generalizing some known families by using a distortion matrix $\Sigma$.

In this study, we discuss a method to construct a new class of bivariate asymmetric copulas based on products of symmetric (sometimes asymmetric) copulas with powered arguments. Then we would like to determine if the proposed construction can offer an added value for modeling asymmetric data. This construction is based on the result of Durante (2009). Our proposal is actually an extension of Durante (2009) for a wide range of copulas which includes some newly constructed copulas in addition to all copula families available in the current literature. With these newly constructed copulas, we investigate dependence properties and measure of association between random variables. We consider the result of Mukherjee et al. (2015) in which they obtained meaningful results of the two non-parametric measures of association between two random variables, Spearman's rho $(\rho)$ and Kendall's tau $(\tau)$, with the asymmetric copula family. In addition, to test the symmetry of data for using bivariate copulas, we use Cramér-von Mises criterion suggested by Genest et al. (2012). Moreover, the estimation of hyper-parameters by the maximum likelihood method are discussed.

This paper is organized as follows. Section 2 contains some basic concepts of copulas and the dependence structure by calculating Spearman's rho and Kendall's tau using asymmetric copulas. In Section 3, we introduce Fourier copula and new class of bivariate asymmetric copulas. Goodnessof-fit of the proposed asymmetric copulas is introduced in Section 4. Test of symmetry for bivariate case and the maximum likelihood estimation of hyper-parameters for the constructed copulas are discussed in Sections 5 and 6, respectively. Section 7 shows the illustrative data analysis for the proposed asymmetric copula models with two real data. Finally, the discussion and conclusion are presented in Section 8.

\section{Definition and preliminary}

In this section we recall some definitions and results that are necessary to understand a (bivariate) copula. A copula is a multivariate distribution function defined on $\mathbb{I}^{n}$, where $\mathbb{I}:=[0,1]$, with uniformly distributed marginals. In this paper, we focus on bivariate copulas.

Definition 1. A bivariate copula is a function $C: \mathbb{I}^{2} \rightarrow \mathbb{I}$, which satisfies the following properties:

(P1) $C(0, v)=C(u, 0)=0, \quad \forall u, v \in \mathbb{I}$

(P2) $C(1, u)=C(u, 1)=u, \quad \forall u \in \mathbb{I}$

(P3) $C$ is 2-increasing, i.e., $\forall u_{1}, u_{2}, v_{1}, v_{2} \in \mathbb{I}$ with $u_{1} \leq u_{2}, v_{1} \leq v_{2}$,

$$
C\left(u_{2}, v_{2}\right)+C\left(u_{1}, v_{1}\right)-C\left(u_{1}, v_{2}\right)-C\left(u_{2}, v_{1}\right) \geq 0 .
$$

The importance of copulas has been growing because of their applications in several fields of research. Their relevance primarily comes from Sklar's Theorem (Sklar, 1959): If X and Y are two continuous random variables with joint distribution function $H$ and marginal distribution functions $F$ and $G$, respectively, then there exists a unique copula $C$ such that $H(x, y)=C(F(x), G(y))$ for all 
$(x, y) \in \mathbb{R}^{2}$ and conversely, given a copula $C$ and two univariate distribution functions $F$ and $G$, the function $H$ defined above is a joint distribution function with margins $F$ and $G$. Sklar's Theorem clarifies the role that copulas play in the relationship between multivariate distribution functions and their univariate margins. A proof of this theorem can be found in Schweizer and Sklar (1983).

Definition 2. Suppose $X$ and $Y$ are two random variables with marginal distribution functions $F$ and $G$, respectively. Then Spearman's rho is the ordinary (Pearson) correlation coefficient of the transformed random variables $F(X)$ and $G(Y)$, while Kendall's tau is the difference between the probability of concordance $\operatorname{Pr}\left[\left(X_{1}-X_{2}\right)\left(Y_{1}-Y_{2}\right)>0\right]$ and the probability of discordance $\operatorname{Pr}\left[\left(X_{1}-X_{2}\right)\left(Y_{1}-Y_{2}\right)<0\right]$ for two independent pairs $\left(X_{1}, Y_{1}\right)$ and $\left(X_{2}, Y_{2}\right)$ of observations drawn from the distribution.

In terms of dependence properties, Spearman's rho is a measure of average quadrant dependence, while Kendall's tau is a measure of average likelihood ratio dependence (see Nelsen (2006) for details). If $X$ and $Y$ are two continuous random variables with copula $C$, then Spearman's rho and Kendall's tau of $X$ and $Y$ are given by,

$$
\begin{aligned}
& \rho=12 \iint_{\mathbb{I}^{2}} C(u, v) d u d v-3, \\
& \tau=4 \iint_{\mathbb{I}^{2}} C(u, v) d C(u, v)-1 .
\end{aligned}
$$

Definition 3. A copula $C$ is called absolutely continuous if, when considered as a joint distribution function, $C(u, v)$ has a joint density function given by $c(u, v):=\partial^{2} C /(\partial u \partial v)$ and in that case $d C(u, v)=$ $\partial^{2} C /(\partial u \partial v) d u d v$.

Denoting $c(u, v)-1$ as $h(u, v)$, the following theorem gives a characterization of absolutely continuous copulas (De la Peña et al., 2006).

Theorem 1. A function $C: \mathbb{I}^{2} \rightarrow \mathbb{I}$ is an absolutely bivariate copula only if there exists a function $h: \mathbb{I}^{2} \rightarrow \mathbb{I}$, satisfying the following conditions,

1. Integrability: $\iint_{\mathbb{I}^{2}}|h(x, y)| d x d y<\infty$,

2. Degeneracy: $\int_{\mathbb{I}} h(x, \xi) d \xi=\int_{\mathbb{I}} h(\xi, y) d \xi=0 \forall x, y \in \mathbb{I}$,

3. Positive Definiteness: $h(x, y) \geq-1 \forall(x, y) \in \mathbb{I}^{2}$,

and such that $C(u, v)=\int_{0}^{v} \int_{0}^{u} 1+h(x, y) d x d y$.

A copula $C$ is called symmetric if $C(u, v)=C(v, u)$ for all $u, v \in \mathbb{I}$, otherwise asymmetric. Let us denote the independent copula as $\Pi(u, v):=u v$. In addition, the new asymmetric copulas satisfying all the hypothesis of Theorem 1 were proposed in Mukherjee et al. (2015):

$$
\begin{aligned}
C_{\max }^{\varepsilon}(u, v)= & \Pi(u, v) \\
& +\frac{1}{4}\left(\sqrt{1+4 \varepsilon^{2}}-\sqrt{(1-2 u)^{2}+4 \varepsilon^{2}}\right)\left(\sqrt{1+4 \varepsilon^{2}}-\sqrt{(1-2 v)^{2}+4 \varepsilon^{2}}\right), \\
C_{\min }^{\varepsilon}(u, v)= & \Pi(u, v) \\
& -\frac{1}{4}\left(\sqrt{1+4 \varepsilon^{2}}-\sqrt{(1-2 u)^{2}+4 \varepsilon^{2}}\right)\left(\sqrt{1+4 \varepsilon^{2}}-\sqrt{(1-2 v)^{2}+4 \varepsilon^{2}}\right) .
\end{aligned}
$$


Then corresponding Spearman's rho and Kendall's tau are given by, respectively,

$$
\begin{aligned}
& \rho_{\text {max }}^{\varepsilon}=\frac{3}{4}\left(\sqrt{1+4 \varepsilon^{2}}-4 \varepsilon^{2} \operatorname{coth}^{-1}\left(\sqrt{1+4 \varepsilon^{2}}\right)\right)^{2}, \\
& \rho_{\min }^{\varepsilon}=-\frac{3}{4}\left(\sqrt{1+4 \varepsilon^{2}}-4 \varepsilon^{2} \operatorname{coth}^{-1}\left(\sqrt{1+4 \varepsilon^{2}}\right)\right)^{2}, \\
& \tau_{\max }^{\varepsilon}=\frac{1}{2}\left[1+4 \varepsilon^{2}+4 \varepsilon^{2}\left(\sqrt{1+4 \varepsilon^{2}}-2 \varepsilon^{2} \operatorname{coth}^{-1}\left(\sqrt{1+4 \varepsilon^{2}}\right)\right) \ln \left(\frac{1+2 \varepsilon^{2}-\sqrt{1+4 \varepsilon^{2}}}{2 \varepsilon^{2}}\right)\right], \\
& \tau_{\min }^{\varepsilon}=-\frac{1}{2}\left[1+4 \varepsilon^{2}+4 \varepsilon^{2}\left(\sqrt{1+4 \varepsilon^{2}}-2 \varepsilon^{2} \operatorname{coth}^{-1}\left(\sqrt{1+4 \varepsilon^{2}}\right)\right) \ln \left(\frac{1+2 \varepsilon^{2}-\sqrt{1+4 \varepsilon^{2}}}{2 \varepsilon^{2}}\right)\right] .
\end{aligned}
$$

The optimal values of $\rho$ and corresponding $\tau$ are obtained by letting $\varepsilon \rightarrow 0$. Mukherjee et al. (2015) showed how the values of $\rho$ approach the optimal values as $\varepsilon \rightarrow 0$ and it is clear that $-0.75 \leq \rho \leq 0.75$ and $-0.5 \leq \tau \leq 0.5$.

\section{Construction of asymmetric copulas}

In this section we will first define Fourier copulas (Lowin, 2010) and then construct asymmetric (in general) copulas using the following theorem (see Durante (2009) for details).

Theorem 2. For all $\alpha, \beta \in(0,1)$, and for all copulas $A$ and $B$, the function $C_{\alpha, \beta}: \mathbb{I}^{2} \rightarrow \mathbb{I}$, defined by

$$
C_{\alpha, \beta}(u, v)=A\left(u^{\alpha}, v^{\beta}\right) B\left(u^{\bar{\alpha}}, v^{\bar{\beta}}\right)
$$

is a copula, where $\bar{\alpha}=1-\alpha$ and $\bar{\beta}=1-\beta$.

\subsection{Fourier copula}

It is natural to write the function $h$ in Theorem 1 as a Fourier series as follows

$$
h(x, y)=\sum_{m, n \in \mathbb{Z}^{0}} \gamma_{m}^{n} \exp (2 \pi i(n x+m y)), \quad \forall(x, y) \in \mathbb{I}^{2},
$$

where $\mathbb{Z}^{0}=\mathbb{Z} \backslash\{0\}$ and $\sum_{m, n \in \mathbb{Z}^{0}}\left|\gamma_{m}^{n}\right|<\infty$ with $\gamma_{-m}^{n}=\overline{\gamma_{m}^{-n}}, \forall n, m \in \mathbb{Z}^{0}$. The latter condition guarantees that $h$ is real valued. Then the integrability and degeneracy of $h$ are clear. For positive definiteness, suppose $\gamma_{m}^{n}$ are chosen so that $h(u, v) \geq-1$ for all $u, v \in \mathbb{I}$, then the copula generated by $h$, defined by

$$
\begin{aligned}
C_{F}(u, v) & =\Pi(u, v)+\int_{t=0}^{v} \int_{s=0}^{u} h(s, t) d s d t \\
& =\Pi(u, v)+\int_{t=0}^{v} \int_{s=0}^{u} \sum_{m, n \in \mathbb{Z}^{0}} \gamma_{m}^{n} \exp (2 \pi i(n x+m y)) d s d t \\
& =\Pi(u, v)-\frac{1}{4 \pi^{2}} \sum_{m, n \in \mathbb{Z}^{0}} \frac{\gamma_{m}^{n}}{m n}\left(e^{2 \pi i n u}-1\right)\left(e^{2 \pi i m v}-1\right)
\end{aligned}
$$

is called a Fourier copula, which was apparently introduced by Ibragimov (2009). It is sufficient that if

$$
\sum_{n, m \in \mathbb{N}}\left|\gamma_{m}^{n}\right|+\left|\gamma_{m}^{-n}\right| \leq \frac{1}{2},
$$




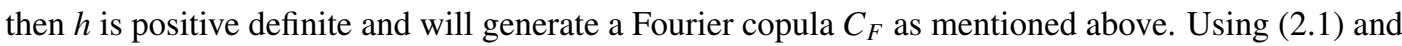

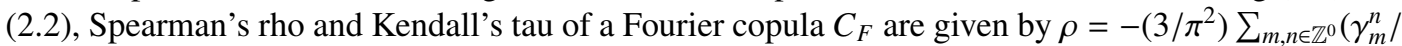

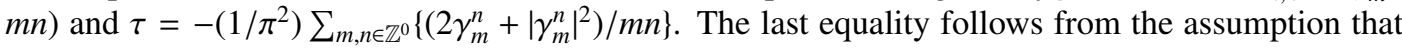

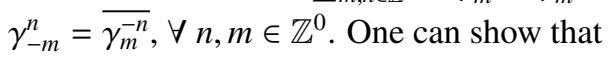

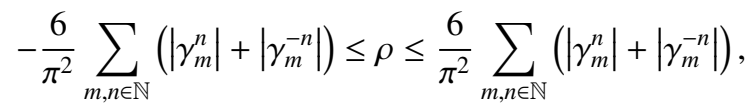

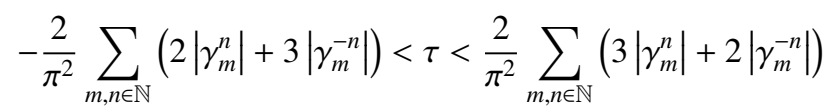

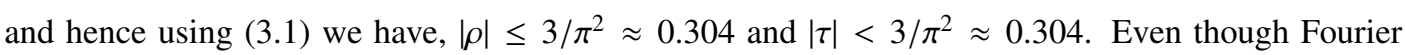

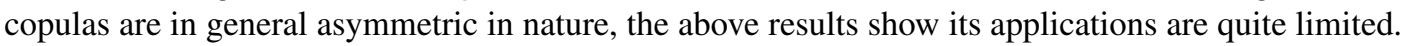

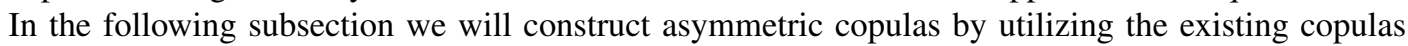

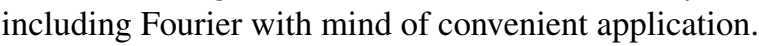

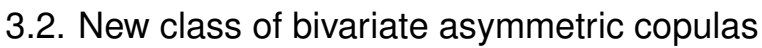

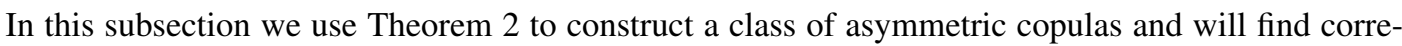

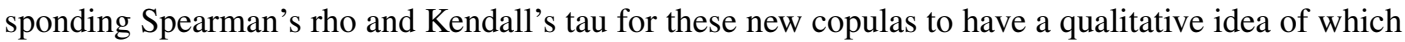

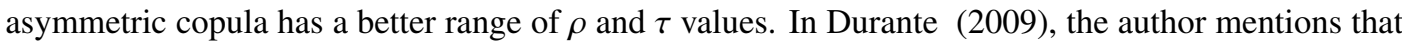

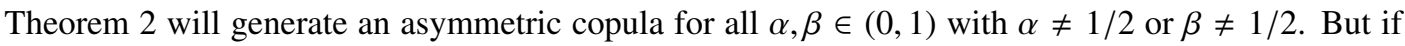

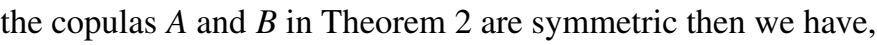

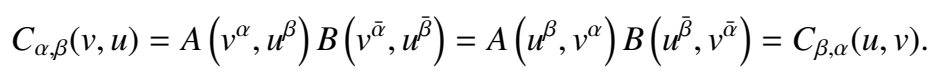

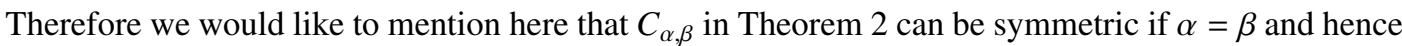

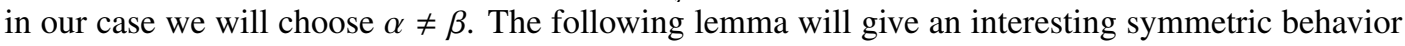

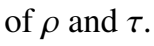

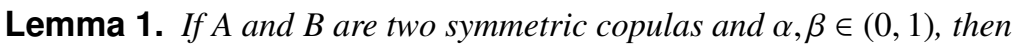

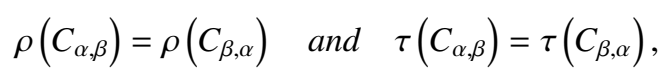

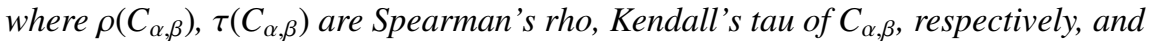

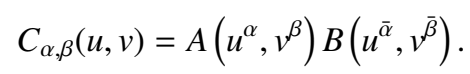

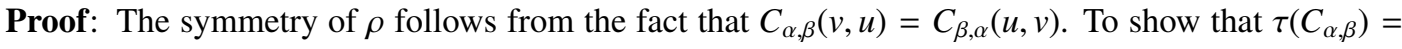

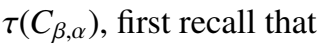

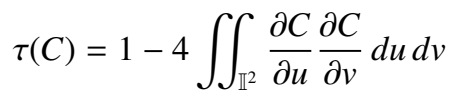

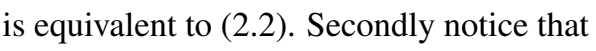

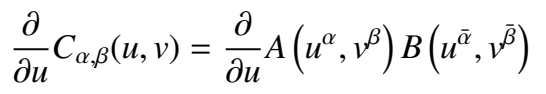

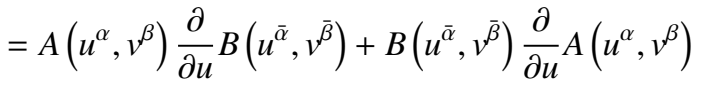

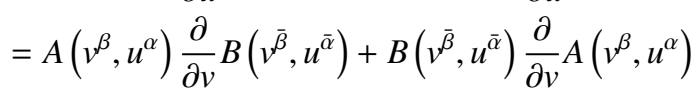


Table 1: Nine basic Copula Functions used in this study to construct bivariate asymmetric copulas

\begin{tabular}{cl}
\hline \hline Copula name \\
\hline Fourier & \multicolumn{1}{c}{ Copula function } \\
\hline Max & $C 2(u, v):=C_{F}(u, v):=C_{\max }^{\varepsilon_{1}}(u, v), \quad \varepsilon_{1}>0$ \\
\hline Min & $C 3(u, v):=C_{\min }^{\varepsilon_{2}}(u, v), \quad \varepsilon_{2}>0$ \\
\hline Independent & $C 4(u, v):=\Pi(u, v)=u v$ \\
\hline FGM & $C 5(u, v):=\operatorname{FGM}\left(u, v, \theta_{1}\right)=u v+\theta_{1} u v(1-u)(1-v), \quad \theta_{1} \in(-1,1]$ \\
\hline Clayton & $C 6(u, v):=\operatorname{Clayton}\left(u, v, \theta_{2}\right)=\left(u^{-\theta_{2}}+v^{\left.-\theta_{2}-1\right)^{-1 / \theta_{2}}, \theta_{2} \in(0, \infty)}\right.$ \\
\hline Frank & $C 7(u, v):=\operatorname{Frank}\left(u, v, \theta_{3}\right)=-\frac{1}{\theta_{3}} \log \left[1+\frac{\left(e^{-\theta_{3} u}-1\right)\left(e^{-\theta_{3} v}-1\right)}{e^{-\theta_{3}}-1}, \theta_{3} \in \mathbb{R} \backslash\{0\}\right.$ \\
\hline Gumbel & $C 8(u, v):=\operatorname{Gumbel}\left(u, v, \theta_{4}\right)=\exp \left[-\left((-\log u)^{\theta_{4}}+(-\log v)^{\theta_{4}}\right)\right.$ \\
\hline AMH & $C 9(u, v):=\operatorname{AMH}\left(u, v, \theta_{5}\right)=\frac{\theta_{4} \geq 1}{1-\theta_{5}(1-u)(1-v)}, \theta_{5} \in(-1,1]$ \\
\hline \hline
\end{tabular}

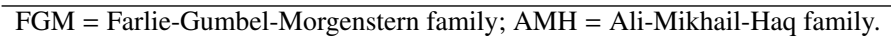

$$
\begin{aligned}
& =\frac{\partial}{\partial \nu} A\left(v^{\beta}, u^{\alpha}\right) B\left(v^{\bar{\beta}}, u^{\bar{\alpha}}\right) \\
& =\frac{\partial}{\partial v} C_{\beta, \alpha}(v, u) .
\end{aligned}
$$

Hence we have

$$
\begin{aligned}
\tau\left(C_{\alpha, \beta}\right) & =1-4 \iint_{\mathbb{I}^{2}} \frac{\partial C_{\alpha, \beta}}{\partial u} \frac{\partial C_{\alpha, \beta}}{\partial v} d u d v \\
& =1-4 \iint_{\mathbb{I}^{2}} \frac{\partial C_{\beta, \alpha}}{\partial v} \frac{\partial C_{\beta, \alpha}}{\partial u} d u d v \\
& =\tau\left(C_{\beta, \alpha}\right) .
\end{aligned}
$$

For convenience we adopt the following notations, for $j=1,2, \ldots$, copulas $C j$ are defined in Table 1. The list of copulas in Table 1 is considered in this study. We define the set of parameters $\psi$ and the copulas that arise from Theorem 2 as,

$$
\begin{aligned}
\psi & :=\left\{\gamma_{m}^{n}, \varepsilon_{1}, \varepsilon_{2}, \theta_{1}, \theta_{2}, \theta_{3}, \theta_{4}, \theta_{5}\right\} \\
C j k_{\alpha, \beta}(u, v) & :=C j\left(u^{\alpha}, v^{\beta}\right) C k\left(u^{\bar{\alpha}}, v^{\bar{\beta}}\right), \quad \text { for } j, k=1,2, \ldots, 9 .
\end{aligned}
$$

Notice that $C j k_{\alpha, \beta} \equiv C k j_{\bar{\alpha}, \bar{\beta}}$.

For $\psi=\left\{0.5 \delta_{m}^{n}\left(\delta_{m}^{1}+\delta_{n}^{-1}\right), 0.01,0.01,1,20,30,20,1\right\}$, where $\delta_{m}^{n}$ is the Kronecker delta, we have calculated (Mathematica code and the results of many other different cases can be found at http://goo.gl/ plkJ7). Spearman's rho and Kendall's tau of the copulas $C j k_{\alpha, \beta}$, for $j, k=1,2, \ldots, 9 ; j<k$ with different $\alpha, \beta$ values. In general, our results show that $\rho, \tau$ values stay away from zero if $(\alpha, \beta) \approx$ $(0,0)$ or/and $(1,1)$. For instance, we would like to mention $\rho, \tau$ values for two copulas $C 12_{\alpha, \beta}$ and $C 17_{\alpha, \beta}$ (Table 2 and Table 3 ).

Figures 1 and 2 clearly show that the contour plots of $C 12_{\alpha, \beta}$ and $C 17_{\alpha, \beta}$ are asymmetric. In this article, the authors just showed the contour plots of two asymmetric copulas, but readers can download the Mathematica code from the linked website and reproduce the contour plots of the other remaining asymmetric copulas. So depending on the readers' provided data, readers can choose one of 
Table 2: $\rho, \tau$ values (correct up to 2 decimal places) for $C 12_{\alpha, \beta}$

\begin{tabular}{|c|c|c|c|c|c|c|c|c|c|}
\hline \multirow{2}{*}{$\alpha$} & & \multicolumn{8}{|c|}{$\beta$} \\
\hline & & 0.1 & 0.2 & $\overline{0.3}$ & 0.4 & 0.5 & 0.6 & 0.7 & 0.8 \\
\hline \multirow{2}{*}{0.2} & $\rho$ & 0.62 & & & & & & & \\
\hline & $\tau$ & 0.41 & & & & & & & \\
\hline \multirow{2}{*}{0.3} & $\rho$ & 0.56 & 0.51 & & & & & & \\
\hline & $\tau$ & 0.37 & 0.34 & & & & & & \\
\hline \multirow{2}{*}{0.4} & $\rho$ & 0.49 & 0.44 & 0.38 & & & & & \\
\hline & $\tau$ & 0.32 & 0.29 & 0.25 & & & & & \\
\hline \multirow{2}{*}{0.5} & $\rho$ & 0.41 & 0.36 & 0.30 & 0.23 & & & & \\
\hline & $\tau$ & 0.27 & 0.24 & 0.20 & 0.15 & & & & \\
\hline \multirow{2}{*}{0.6} & $\rho$ & 0.33 & 0.28 & 0.21 & 0.15 & 0.08 & & & \\
\hline & $\tau$ & 0.22 & 0.18 & 0.14 & 0.10 & 0.05 & & & \\
\hline \multirow{2}{*}{0.7} & $\rho$ & 0.24 & 0.19 & 0.13 & 0.07 & 0.01 & -0.05 & & \\
\hline & $\tau$ & 0.16 & 0.13 & 0.09 & 0.04 & 0.00 & -0.04 & & \\
\hline \multirow{2}{*}{0.8} & $\rho$ & 0.15 & 0.10 & 0.05 & -0.00 & -0.06 & -0.11 & -0.15 & \\
\hline & $\tau$ & 0.10 & 0.07 & 0.03 & -0.01 & -0.05 & -0.08 & -0.12 & \\
\hline \multirow{2}{*}{0.9} & $\rho$ & 0.06 & 0.02 & -0.02 & -0.07 & -0.12 & -0.16 & -0.20 & -0.23 \\
\hline & $\tau$ & 0.04 & 0.01 & -0.02 & -0.05 & -0.09 & -0.12 & -0.15 & -0.18 \\
\hline
\end{tabular}

Table 3: $\rho, \tau$ values (correct up to 2 decimal places) for $C 17_{\alpha, \beta}$

\begin{tabular}{|c|c|c|c|c|c|c|c|c|c|}
\hline \multirow{2}{*}{$\alpha$} & & \multicolumn{8}{|c|}{$\beta$} \\
\hline & & 0.1 & 0.2 & 0.3 & 0.4 & 0.5 & 0.6 & 0.7 & 0.8 \\
\hline \multirow{2}{*}{0.2} & $\rho$ & 0.81 & & & & & & & \\
\hline & $\tau$ & 0.67 & & & & & & & \\
\hline \multirow{2}{*}{0.3} & $\rho$ & 0.73 & 0.68 & & & & & & \\
\hline & $\tau$ & 0.59 & 0.54 & & & & & & \\
\hline \multirow{2}{*}{0.4} & $\rho$ & 0.65 & 0.60 & 0.54 & & & & & \\
\hline & $\tau$ & 0.51 & 0.47 & 0.41 & & & & & \\
\hline \multirow{2}{*}{0.5} & $\rho$ & 0.57 & 0.52 & 0.46 & 0.38 & & & & \\
\hline & $\tau$ & 0.43 & 0.39 & 0.34 & 0.28 & & & & \\
\hline \multirow{2}{*}{0.6} & $\rho$ & 0.47 & 0.42 & 0.36 & 0.30 & 0.23 & & & \\
\hline & $\tau$ & 0.35 & 0.31 & 0.26 & 0.21 & 0.16 & & & \\
\hline \multirow{2}{*}{0.7} & $\rho$ & 0.36 & 0.32 & 0.27 & 0.21 & 0.15 & 0.08 & & \\
\hline & $\tau$ & 0.26 & 0.23 & 0.19 & 0.14 & 0.09 & 0.05 & & \\
\hline \multirow{2}{*}{0.8} & $\rho$ & 0.25 & 0.21 & 0.16 & 0.11 & 0.06 & 0.00 & -0.05 & \\
\hline & $\tau$ & 0.17 & 0.14 & 0.11 & 0.07 & 0.03 & -0.01 & -0.05 & \\
\hline \multirow{2}{*}{0.9} & $\rho$ & 0.12 & 0.09 & 0.04 & 0.00 & -0.05 & -0.09 & -0.13 & -0.17 \\
\hline & $\tau$ & 0.08 & 0.06 & 0.02 & -0.01 & -0.05 & -0.08 & -0.11 & -0.15 \\
\hline
\end{tabular}

the proposed asymmetric copula by looking at the contour plots of all proposed asymmetric copulas. Figure 3 is scatter plots of random numbers generated from the nine basic copulas. Figure 4 is scatter plots of random numbers generated from some of the constructed asymmetric copulas. These figures may be helpful to choose which copula will be appropriate to fit the given data well.

\section{Estimation and goodness-of-fit}

\subsection{Fitting copulas to data}

We assume that we have a random sample $\mathbf{X}_{1}, \ldots, \mathbf{X}_{n}$ from a d-dimensional cumulative distribution function (CDF) $F$ with continuous marginal CDFs $F_{1}, \ldots, F_{d}$. Hence, $F$ has the unique representation, $F\left(x_{1}, \ldots, x_{d}\right)=C\left[F_{1}\left(x_{1}\right), \ldots, F_{d}\left(x_{d}\right)\right]$, by Sklar's Theorem. Let $\hat{F}_{1}, \ldots, \hat{F}_{d}$ denote the rescaled empirical CDFs computed from the data as that for every $j \in\{1, \ldots, d\}, \hat{F}_{j}(x)=\{1 /(n+1)\} \sum_{i=1}^{n} \mathbf{1}\left(X_{i, j}\right.$ 


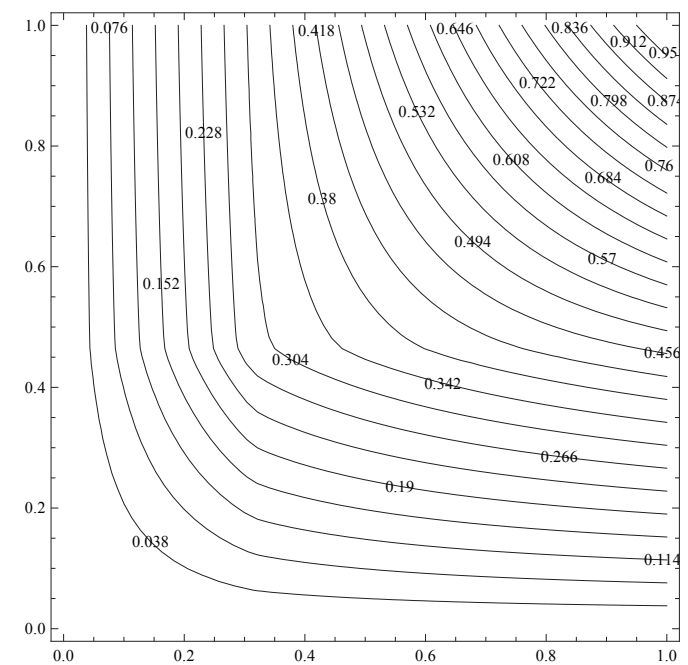

Figure 1: Contour plot of $C 12_{\alpha, \beta}$ with $\alpha=0.4, \beta=0.1$.

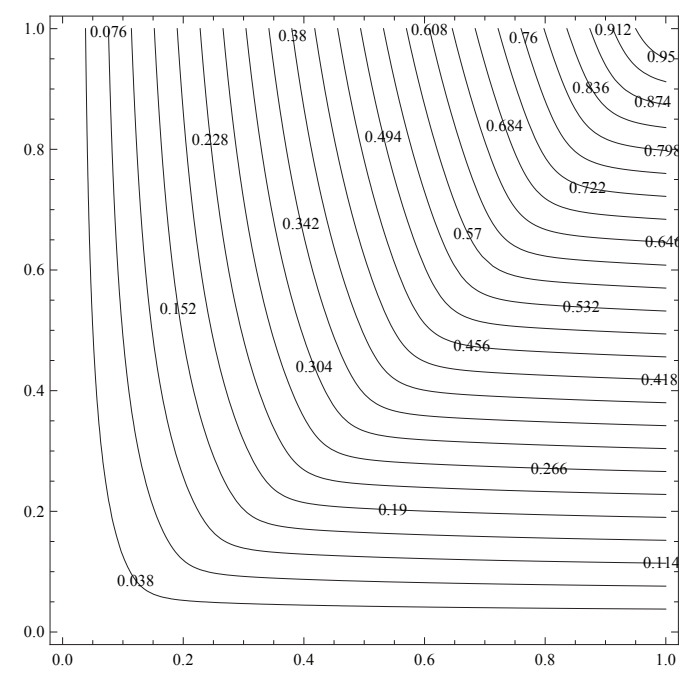

Figure 2: Contour plot of $C 17_{\alpha, \beta}$ with $\alpha=0.1, \beta=0.4$.

$\leq x$ ). The rescaled empirical CDFs differ from the usual empirical CDF by the use of denominator $n+1$ rather than $n$. This guarantees that the pseudo-observations lie strictly in the interior of $[0,1]^{d}$.

The maximum pseudo-likelihood estimate (MPLE) of $\theta$ is obtained by maximizing the log pseudolikelihood with respect to $\theta$;

$$
\log L\left(\theta ; \hat{\mathbf{U}}_{1}, \ldots, \hat{\mathbf{U}}_{n}\right)=\sum_{i=1}^{n} \log c_{\theta}\left(\hat{\mathbf{U}}_{i}\right),
$$

where $c_{\theta}$ denotes the copula density (Kojadinovic, 2013), and

$$
\hat{\mathbf{U}}_{i}=\left(\hat{U}_{i, 1}, \ldots, \hat{U}_{i, d}\right)=\left(\hat{F}_{1}\left(X_{i, 1}\right), \ldots, \hat{F}_{d}\left(X_{i, d}\right)\right)
$$



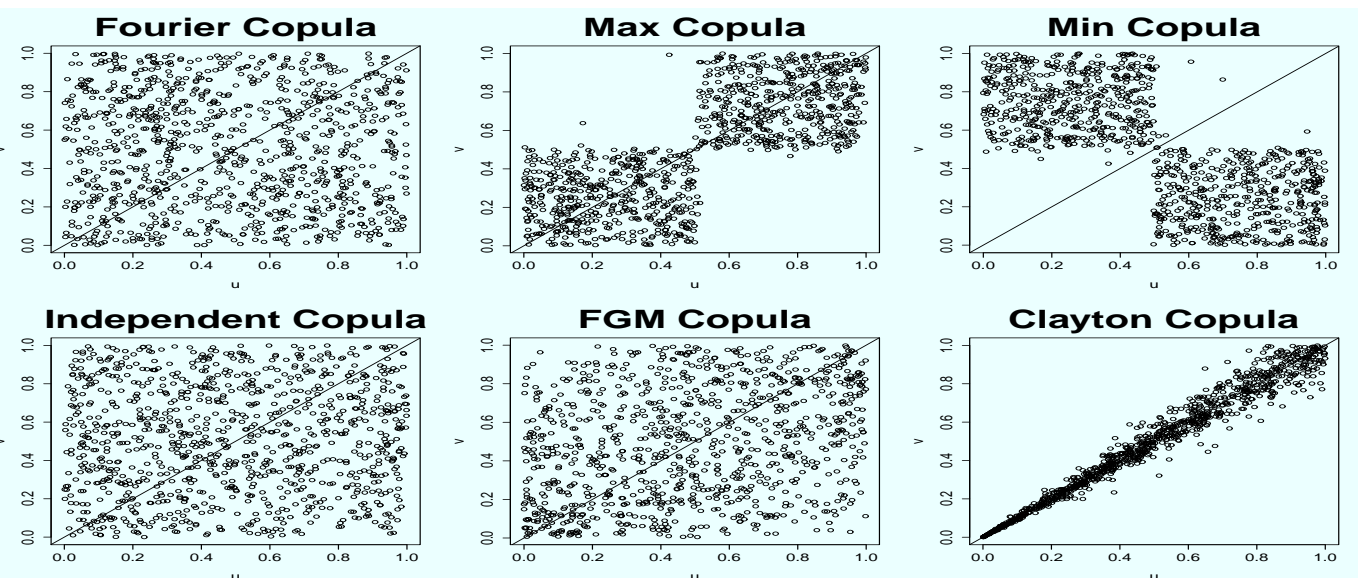

Frank Copula
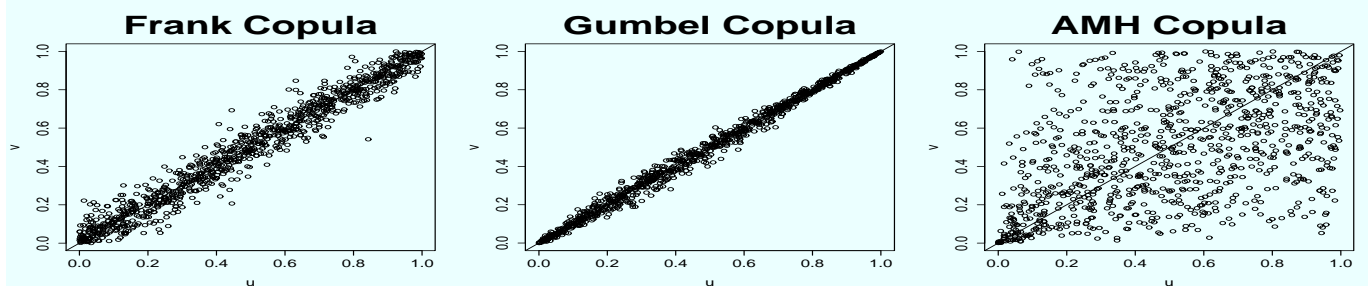

Figure 3: Scatter plots of random numbers generated from the nine basic copulas.
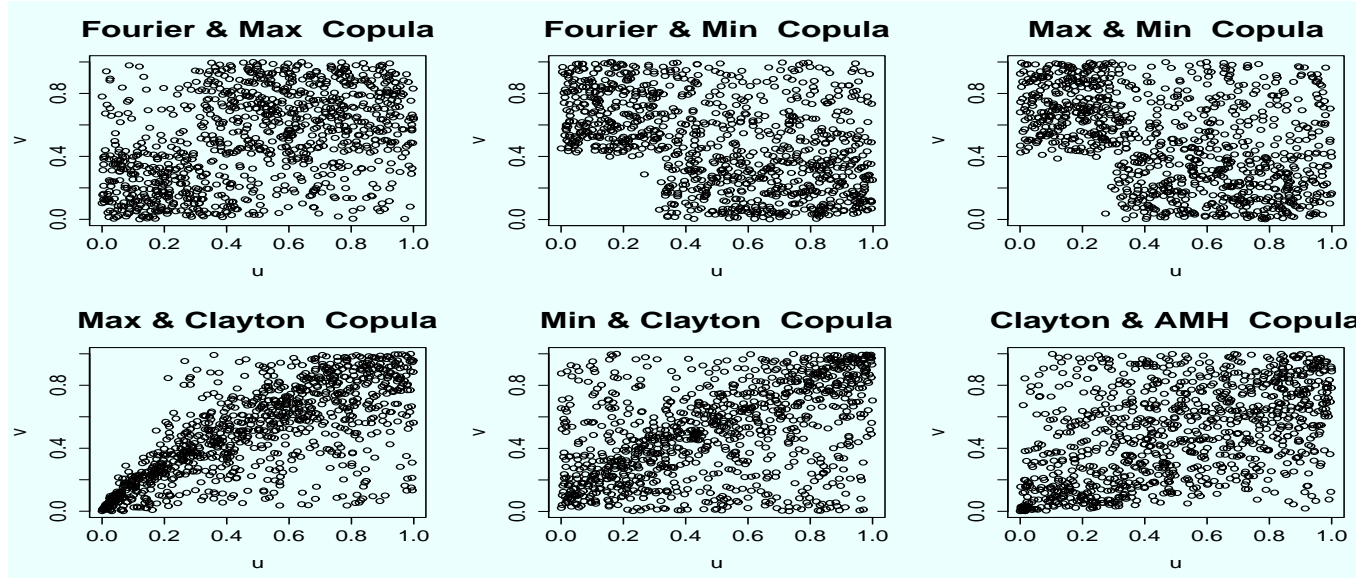

Clayton \& AMH Copula
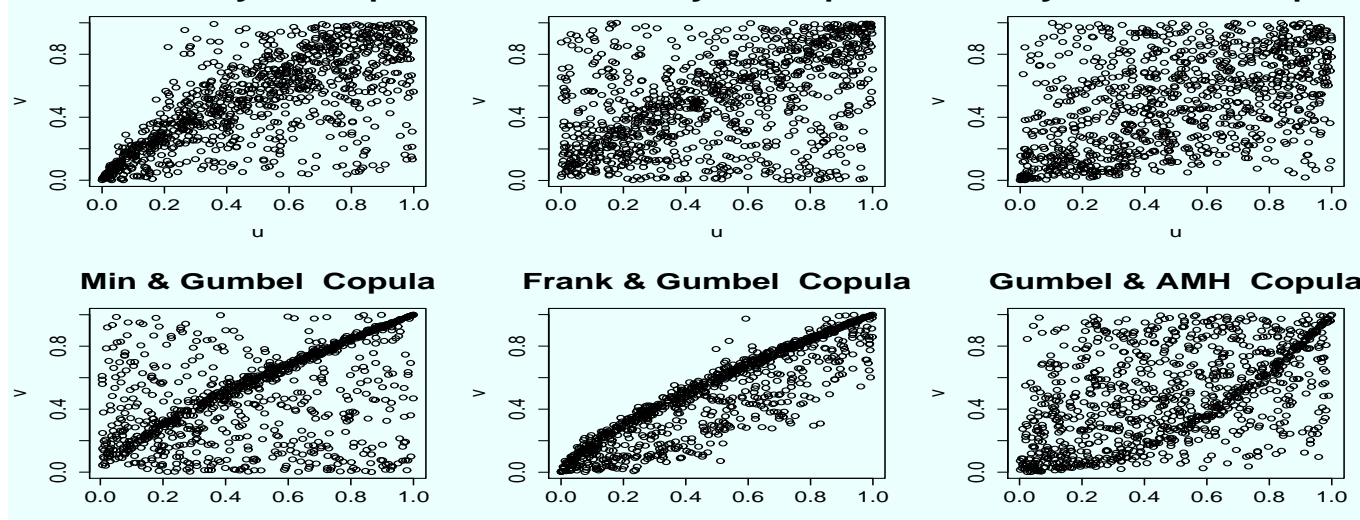

Figure 4: Scatter plots of random numbers generated from the proposed asymmetric copulas. Only nine copula cases are shown here as a sample.

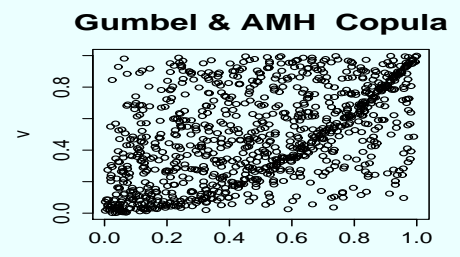




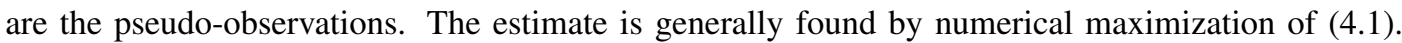

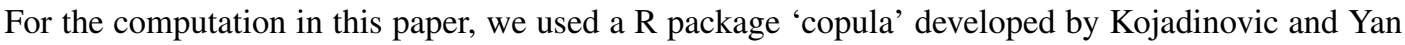

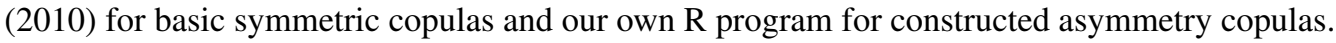

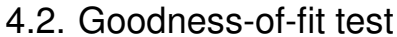

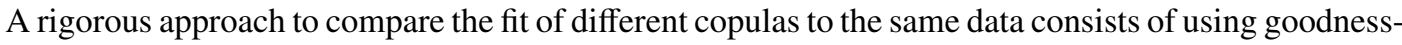

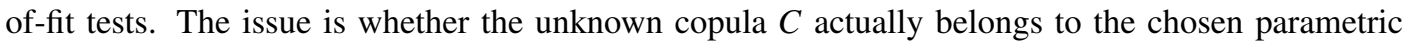

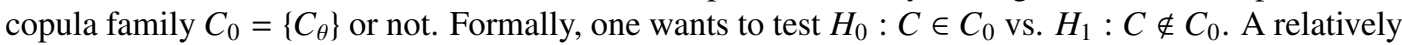

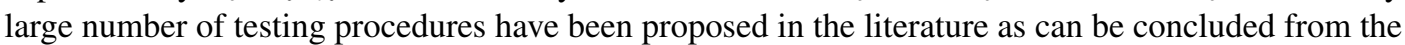

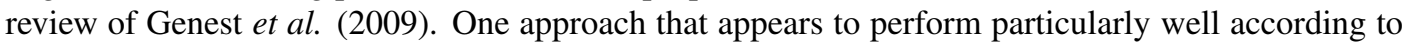

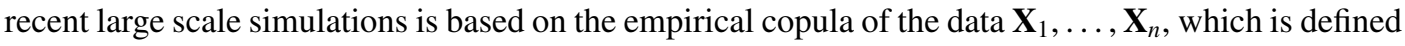

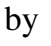

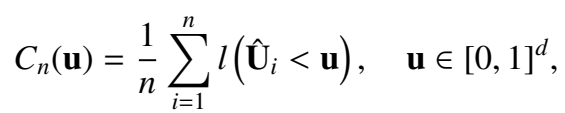

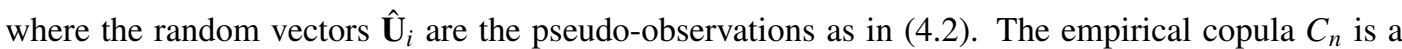

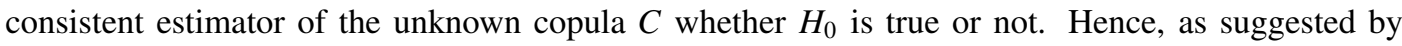

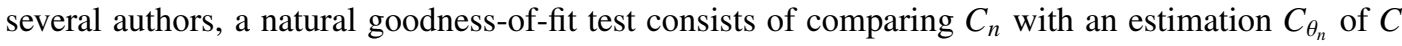

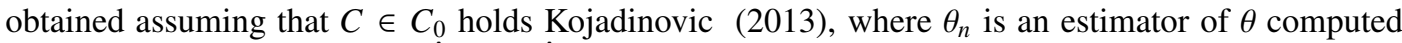

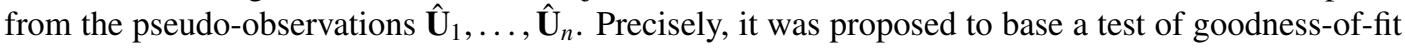

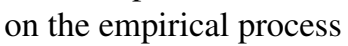

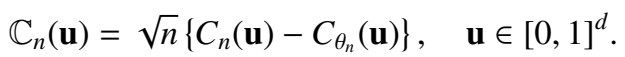

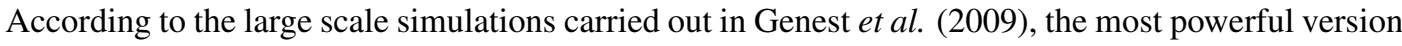

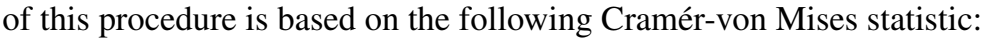

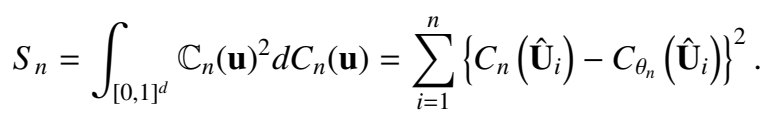

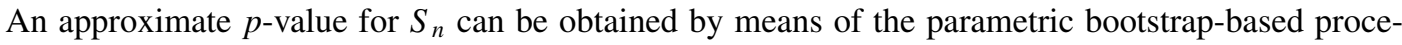

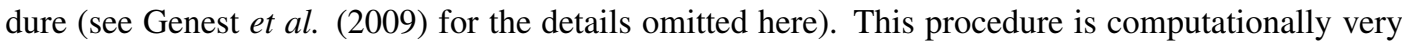

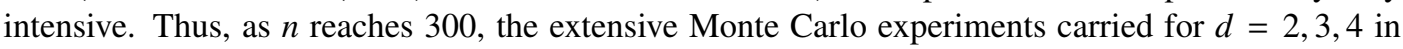

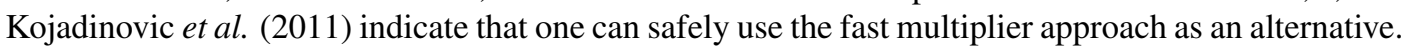

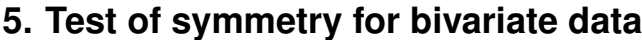

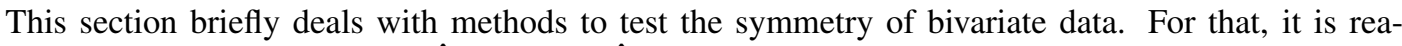

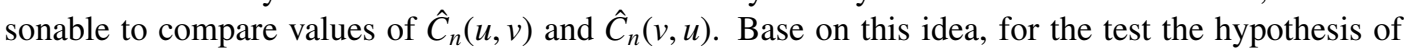

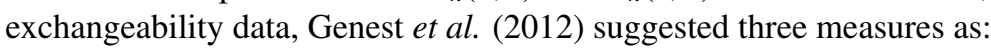

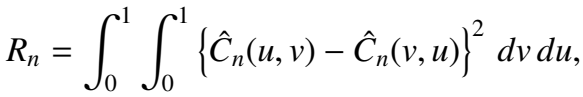

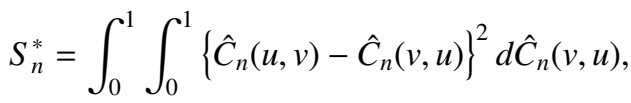

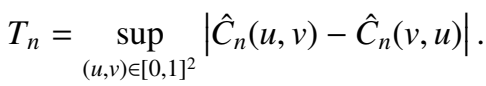




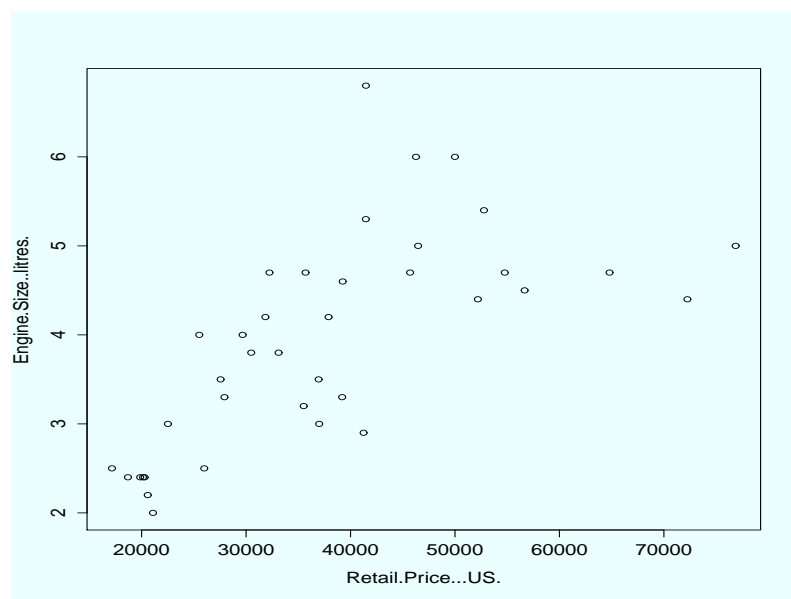

Figure 5: Scatter plot of car rental data.

See also Bouzebda and Cherfi (2012) and Quessy and Bahraoui (2013) for other test procedures for the symmetry of copulas. Nelsen (2007) considered another measure of asymmetry. In this study, we use a Cramér-von Mises statistic $S_{n}^{*}$ as a measure to check asymmetry of bivariate data for the computational convenience. The 'exchTest' function of the 'copula' package in $\mathrm{R}$ program was used for the calculation of the $S_{n}^{*}$.

\section{Estimation of hyper-parameters in constructed asymmetric copula}

In the previous section, we estimated the parameters of copulas with the powered hyper-parameters $\alpha$ and $\beta$. In this section, we explain how to estimate simultaneously the parameter in copulas as well as the hyper-parameters of constructed asymmetric copula.

By the equation (4.1), the $\log$ pseudo-likelihood of the constructed copula by copulas $C_{1}$ and $C_{2}$ is as:

$$
\log L\left(\theta_{1}, \theta_{2}, \alpha, \beta ;\left(\hat{u}_{1}, \hat{v}_{1}\right), \ldots,\left(\hat{u}_{n}, \hat{v}_{n}\right)\right)=\sum_{i=1}^{n} \log C_{1}\left(u_{1}^{\alpha}, v_{1}^{\beta}\right) C_{2}\left(u_{1}^{\bar{\alpha}}, v_{1}^{\bar{\beta}}\right),
$$

where $\left(\hat{u}_{i}, \hat{v}_{i}\right)$ is $i^{\text {th }}$ pseudo-observation and $\theta_{1}$ and $\theta_{2}$ are the parameters of copulas $C_{1}$ and $C_{2}$, respectively. We estimated the parameters $\theta_{1}, \theta_{2}, \alpha, \beta$ by maximizing (6.1), simultaneously. For this optimization computation, we used a quasi-Newton algorithm with numerical differentiation in a ' $\mathrm{L}$ BFGS-B' method in R function 'optim'.

\section{Real data example}

\subsection{Car rental data}

We consider two datasets to illustrate the usefulness of our proposed asymmetric copulas. The first dataset is car rental data of American new cars and trucks data for sport utility vehicle (SUV) with four wheel drive which is available at Nayland College. Engine size and retail price variables with sample size $n=38$ are considered for this study. Figure 5 is a scatter plot of two variables, engine size and retail price. For the symmetry test on this data, we have $S_{n}^{*}=0.056$ with $p$-value $=0.004$ as described in Section 5, which means the data is not symmetric. 


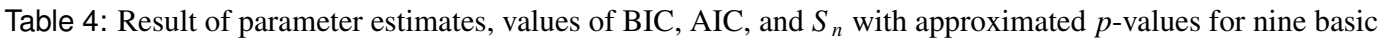

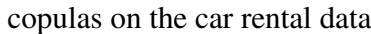

\begin{tabular}{|c|c|c|c|c|c|}
\hline 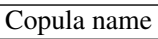 & 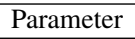 & 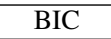 & 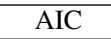 & 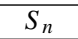 & 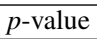 \\
\hline 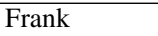 & 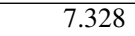 & 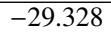 & 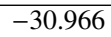 & 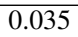 & 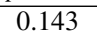 \\
\hline 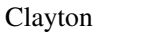 & 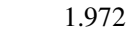 & 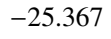 & 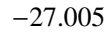 & 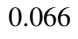 & 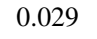 \\
\hline 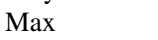 & 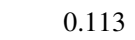 & 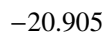 & 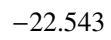 & 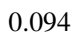 & 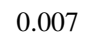 \\
\hline 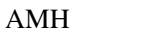 & 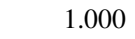 & 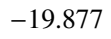 & 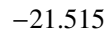 & 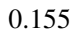 & 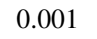 \\
\hline 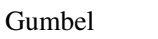 & 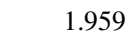 & 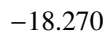 & 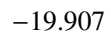 & 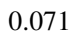 & 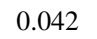 \\
\hline 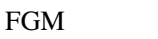 & 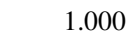 & 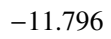 & 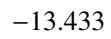 & 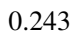 & 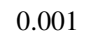 \\
\hline 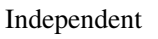 & & 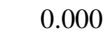 & 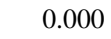 & 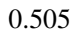 & 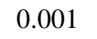 \\
\hline 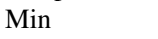 & 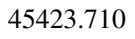 & 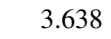 & 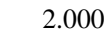 & 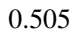 & 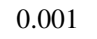 \\
\hline 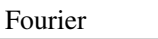 & 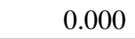 & 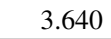 & 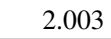 & 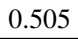 & 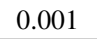 \\
\hline
\end{tabular}

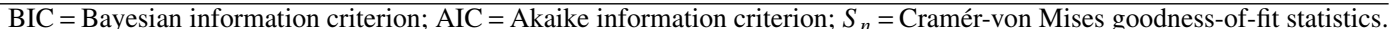

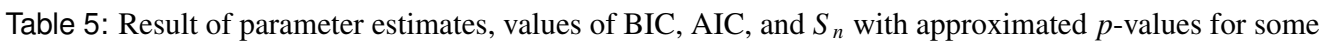

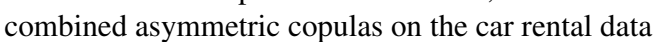

\begin{tabular}{|c|c|c|c|c|c|c|c|c|}
\hline 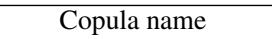 & 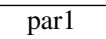 & 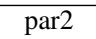 & 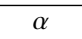 & 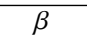 & 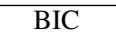 & 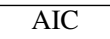 & 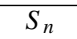 & 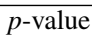 \\
\hline 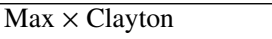 & 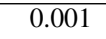 & 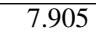 & 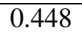 & 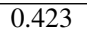 & 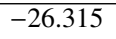 & 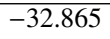 & 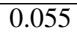 & 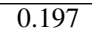 \\
\hline 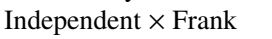 & & 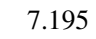 & 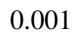 & 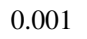 & 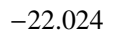 & 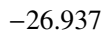 & 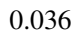 & 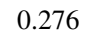 \\
\hline 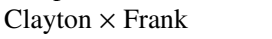 & 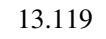 & 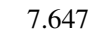 & 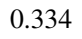 & 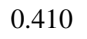 & 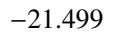 & 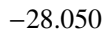 & 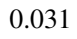 & 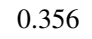 \\
\hline 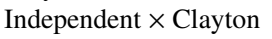 & & 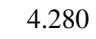 & 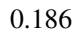 & 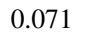 & 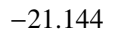 & 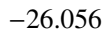 & 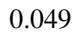 & 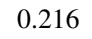 \\
\hline 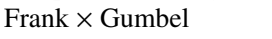 & 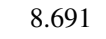 & 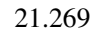 & 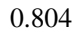 & 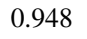 & 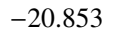 & 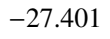 & 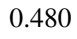 & 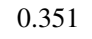 \\
\hline 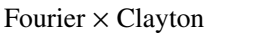 & 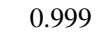 & 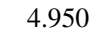 & 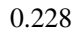 & 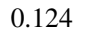 & 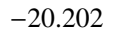 & 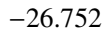 & 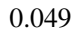 & 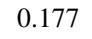 \\
\hline 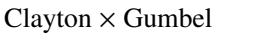 & 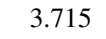 & 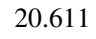 & 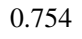 & 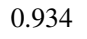 & 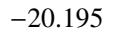 & 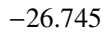 & 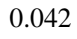 & 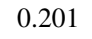 \\
\hline 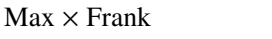 & 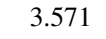 & 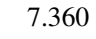 & 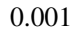 & 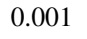 & 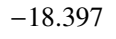 & 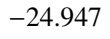 & 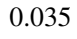 & 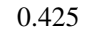 \\
\hline 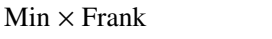 & 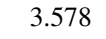 & 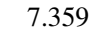 & 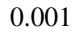 & 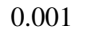 & 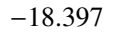 & 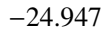 & 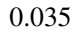 & 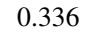 \\
\hline 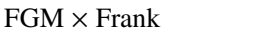 & 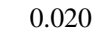 & 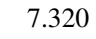 & 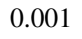 & 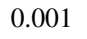 & 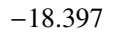 & 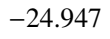 & 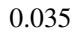 & 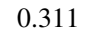 \\
\hline 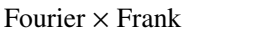 & 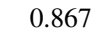 & 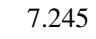 & 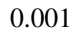 & 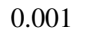 & 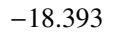 & 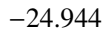 & 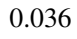 & 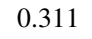 \\
\hline 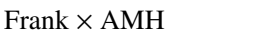 & 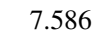 & 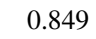 & 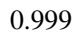 & 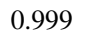 & 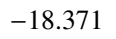 & 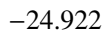 & 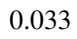 & 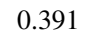 \\
\hline 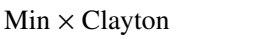 & 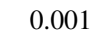 & 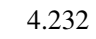 & 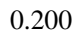 & 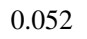 & 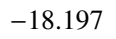 & 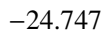 & 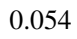 & 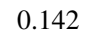 \\
\hline 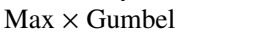 & 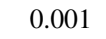 & 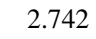 & 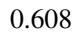 & 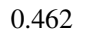 & 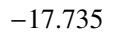 & 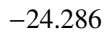 & 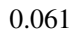 & 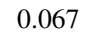 \\
\hline 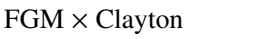 & 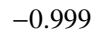 & 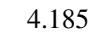 & 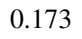 & 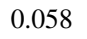 & 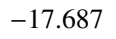 & 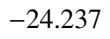 & 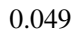 & 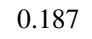 \\
\hline 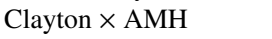 & 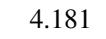 & 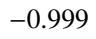 & 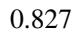 & 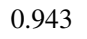 & 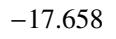 & 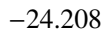 & 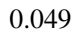 & 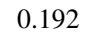 \\
\hline 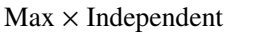 & 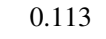 & & 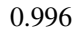 & 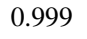 & 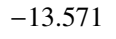 & 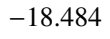 & 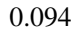 & 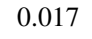 \\
\hline 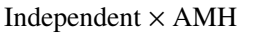 & & 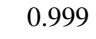 & 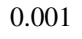 & 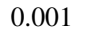 & 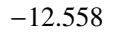 & 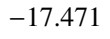 & 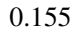 & 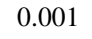 \\
\hline 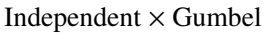 & & 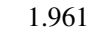 & 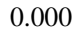 & 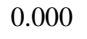 & 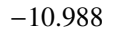 & 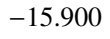 & 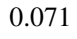 & 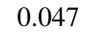 \\
\hline 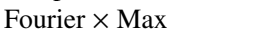 & 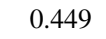 & 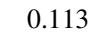 & 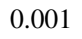 & 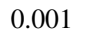 & 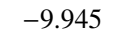 & 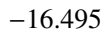 & 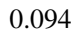 & 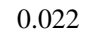 \\
\hline 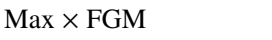 & 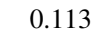 & 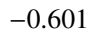 & 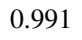 & 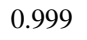 & 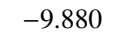 & 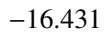 & 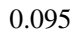 & 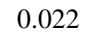 \\
\hline 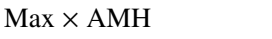 & 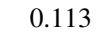 & 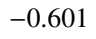 & 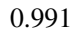 & 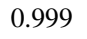 & 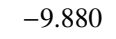 & 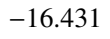 & 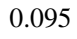 & 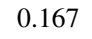 \\
\hline 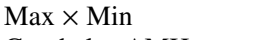 & 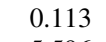 & 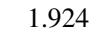 & 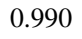 & 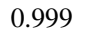 & 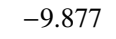 & 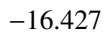 & 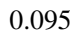 & 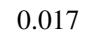 \\
\hline 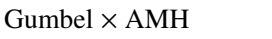 & 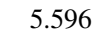 & 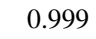 & 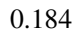 & 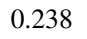 & 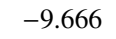 & 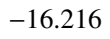 & 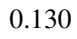 & 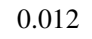 \\
\hline 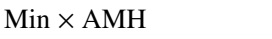 & 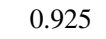 & 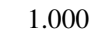 & 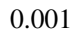 & 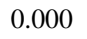 & 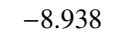 & 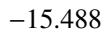 & 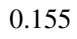 & 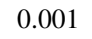 \\
\hline 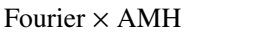 & 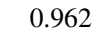 & 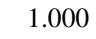 & 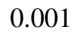 & 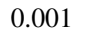 & 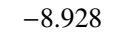 & 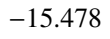 & 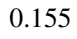 & 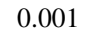 \\
\hline 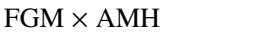 & 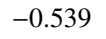 & 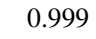 & 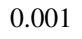 & 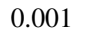 & 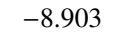 & 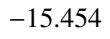 & 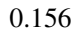 & 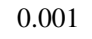 \\
\hline 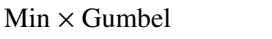 & 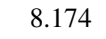 & 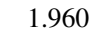 & 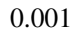 & 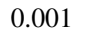 & 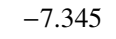 & 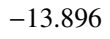 & 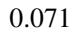 & 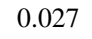 \\
\hline 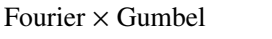 & 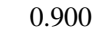 & 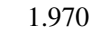 & 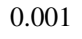 & 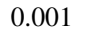 & 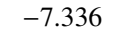 & 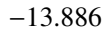 & 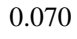 & 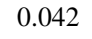 \\
\hline 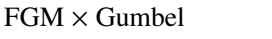 & 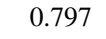 & 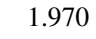 & 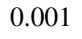 & 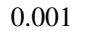 & 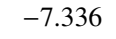 & 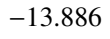 & 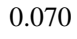 & 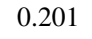 \\
\hline 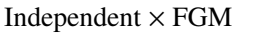 & & 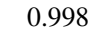 & 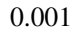 & 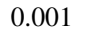 & 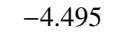 & 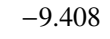 & 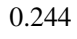 & 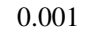 \\
\hline 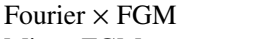 & 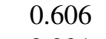 & 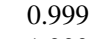 & 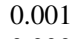 & 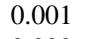 & 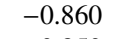 & 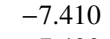 & 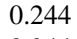 & 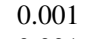 \\
\hline 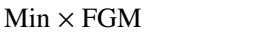 & 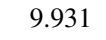 & 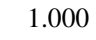 & 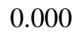 & 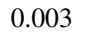 & 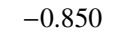 & 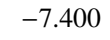 & 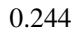 & 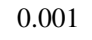 \\
\hline 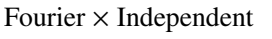 & 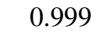 & & 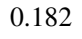 & 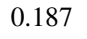 & 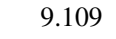 & 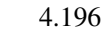 & 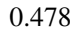 & 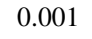 \\
\hline 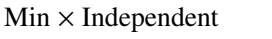 & 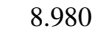 & & 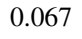 & 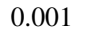 & 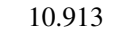 & 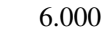 & 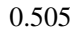 & 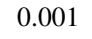 \\
\hline 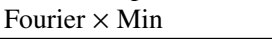 & 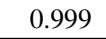 & 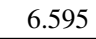 & 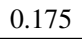 & 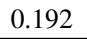 & 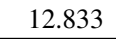 & 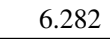 & 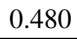 & 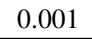 \\
\hline
\end{tabular}

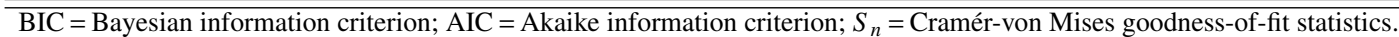



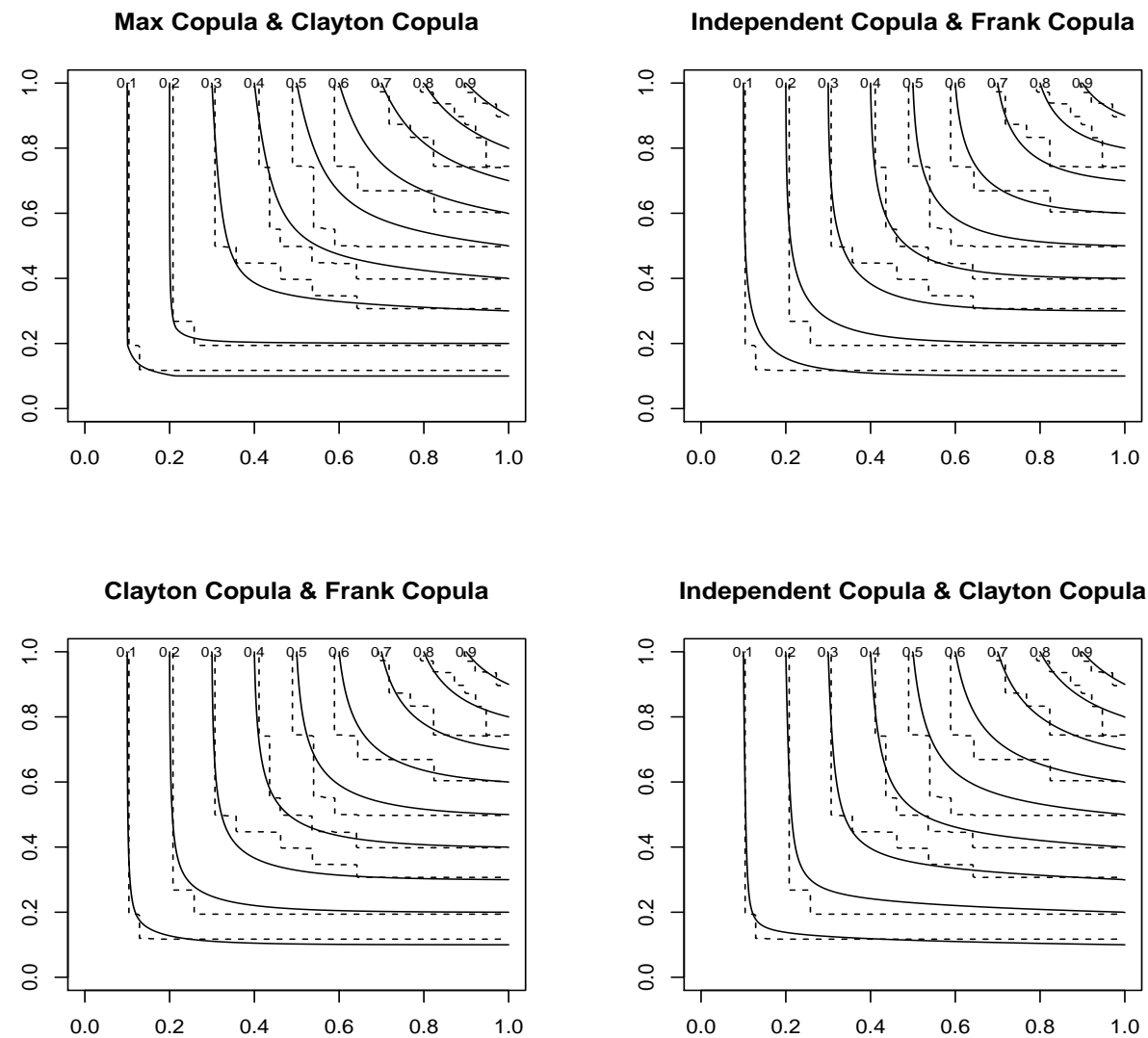

Figure 6: Contour plots of fitted copulas $C_{\theta_{n}}$ (solid line) and empirical copulas $C_{n}$ (dotted line) for the four constructed asymmetric copulas on the car rental data.

Table 4 shows the result of parameter estimates, values of Bayesian information criterion (BIC), values of Akaike information criterion (AIC), and Cramér-von Mises goodness-of-fit statistics $\left(S_{n}\right)$ with approximated $p$-values for nine basic copulas on the car rental data. Only one basic copula fits well in the sense of $5 \%$ level of Cramér-von Mises test: Frank copula. Table 5 shows the result of analysis for the constructed asymmetric copulas. Here $\operatorname{par} 1\left(\theta_{1}\right), \operatorname{par} 2\left(\theta_{2}\right), \alpha$ and $\beta$ are estimated simultaneously by the MPLE as presented in Subsection 4.1 and Section 6. Fifteen combinations show $p$-values greater than 0.05 in Table 5, which means that asymmetric copulas are appropriate model. Figure 6 is the contour plots of empirical copulas $\left(C_{n}\right)$ and fitted copulas $\left(C_{\theta_{n}}\right)$ for four asymmetric copulas: Clayton $\times$ Frank, Fourier $\times$ Frank, Fourier $\times$ Gumbel, and Fourier $\times$ AMH copula.

\subsection{Economic indicators data}

The second datasets is monthly economic indicators of Korea from Jan. 2011 to Aug. 2013, available at (Statistics Korea). Certificate of deposit (CD) rate and interest rate variables with sample size $n=44$ are considered for this study. Figure 7 is a scatter plot of two variables, CD rate and interest rate. For the symmetry test on this data, we have $S_{n}^{*}=0.102$ with $p$-value $=0.008$ as mentioned in 


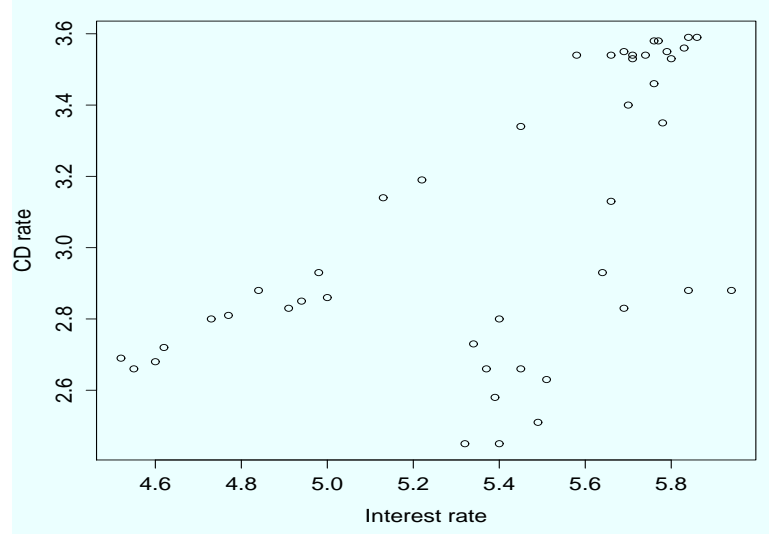

Figure 7: Scatter plot of economic indicators data.

Table 6: Result of parameter estimates, values of BIC, AIC, and $S_{n}$ with approximated $p$-values for nine basic copulas on the economic indicators data

\begin{tabular}{lrrrrr}
\hline \hline Copula name & par & BIC & AIC & $S_{n}$ & $p$-value \\
\hline Max & 0.096 & -26.055 & -27.839 & 0.089 & 0.017 \\
Gumbel & 1.867 & -20.218 & -22.002 & 0.062 & 0.107 \\
Frank & 5.048 & -19.045 & -20.829 & 0.073 & 0.032 \\
FGM & 1.000 & -11.786 & -13.570 & 0.223 & 0.001 \\
AMH & 0.859 & -8.993 & -10.777 & 0.208 & 0.001 \\
Clayton & 0.826 & -6.796 & -8.580 & 0.197 & 0.001 \\
Independent & & 0.000 & 0.000 & 0.462 & 0.001 \\
Min & 87109.604 & 3.784 & 2.000 & 0.462 & 0.001 \\
Fourier & 0.000 & 3.802 & 2.017 & 0.462 & 0.001 \\
\hline \hline
\end{tabular}

BIC = Bayesian information criterion; AIC = Akaike information criterion; $S_{n}=$ Cramér-von Mises goodness-of-fit statistics

Section 5, which means the data is not symmetric.

Table 6 shows the result of parameter estimates, values of BIC, AIC, and $S_{n}$ with approximated $p$-values for nine basic copulas on the car rental data. Only one basic copula fits well in the sense of 5\% level of Cramér-von Mises test: Gumbel copula. Table 7 shows the result of analysis for the constructed asymmetric copulas. Fifteen combinations show $p$-values greater than 0.05 in Table 7 , which means that asymmetric copulas are appropriate model. Figure 8 is the contour plots of empirical copulas $\left(C_{n}\right)$ and fitted copulas $\left(C_{\theta_{n}}\right)$ for four asymmetric copulas: Clayton $\times$ Frank, Fourier $\times$ Frank, Fourier $\times$ Gumbel, and Fourier $\times$ AMH copula.

\section{Conclusion and discussion}

We discussed a new generalized copula family which includes a class of asymmetric copulas as well as all copula families available in the current literature, including Fourier copula. The construction of new asymmetric family is based on and an extension of the result by Durante (2009). With diverse data such as simulated data, car rental data, and economic indicators, we performed parameter estimation by using the maximum pseudo-likelihood estimation method and Cramér-von Mises type of goodness-of-fit tests for the newly constructed asymmetric copula family. For these data, some of the proposed models turned out to be successful whereas the existing copulas were mostly unsuccessful. We thus argue that the proposed construction can offer an added value to model asymmetric bivariate 
Table 7: Result of parameter estimates, values of BIC, AIC, and $S_{n}$ with approximated $p$-values for some combined asymmetric copulas on the economic indicators data

\begin{tabular}{|c|c|c|c|c|c|c|c|c|}
\hline Copula name & par1 & par2 & $\alpha$ & $\beta$ & BIC & AIC & $S_{n}$ & $p$-value \\
\hline Clayton $\times$ Gumbel & 13.478 & 5.576 & 0.605 & 0.172 & -27.187 & -34.324 & 0.044 & 0.236 \\
\hline Frank × Gumbel & 17.132 & 5.471 & 0.598 & 0.166 & -26.130 & -33.267 & 0.043 & 0.221 \\
\hline Independent $\times$ Gumbel & & 5.037 & 0.500 & 0.001 & -25.799 & -31.151 & 0.064 & 0.107 \\
\hline Independent $\times$ Clayton & & 13.916 & 0.483 & 0.080 & -25.585 & -30.938 & 0.072 & 0.082 \\
\hline Fourier $\times$ Clayton & 0.999 & 10.218 & 0.491 & 0.088 & -23.759 & -30.896 & 0.078 & 0.057 \\
\hline Clayton $\times$ Frank & 11.221 & 18.584 & 0.446 & 0.852 & -23.380 & -30.516 & 0.052 & 0.152 \\
\hline Min $\times$ Clayton & 0.001 & 13.566 & 0.474 & 0.067 & -23.172 & -30.309 & 0.078 & 0.052 \\
\hline Fourier $\times$ Gumbel & 0.999 & 4.641 & 0.543 & 0.128 & -23.139 & -30.276 & 0.089 & 0.062 \\
\hline Clayton × AMH & 13.112 & -0.999 & 0.531 & 0.947 & -22.091 & -29.228 & 0.071 & 0.072 \\
\hline FGM $\times$ Gumbel & 0.728 & 4.705 & 0.492 & 0.001 & -22.090 & -29.227 & 0.063 & 0.107 \\
\hline Max × Gumbel & 4.443 & 4.571 & 0.482 & 0.001 & -22.062 & -29.199 & 0.061 & 0.201 \\
\hline Min × Gumbel & 4.446 & 4.569 & 0.481 & 0.001 & -22.062 & -29.199 & 0.061 & 0.152 \\
\hline FGM $\times$ Clayton & -0.999 & 11.810 & 0.466 & 0.046 & -21.995 & -29.132 & 0.072 & 0.067 \\
\hline Gumbel × AMH & 5.108 & 0.876 & 0.499 & 0.999 & -21.979 & -29.116 & 0.064 & 0.112 \\
\hline Max × Clayton & 146.628 & 13.915 & 0.483 & 0.080 & -21.801 & -28.938 & 0.072 & 0.072 \\
\hline Independent $\times$ Frank & & 9.868 & 0.440 & 0.001 & -18.546 & -23.899 & 0.083 & 0.037 \\
\hline Max $\times$ Independent & 0.098 & & 0.999 & 0.999 & -18.457 & -23.810 & 0.090 & 0.012 \\
\hline FGM $\times$ Frank & -0.475 & 10.201 & 0.441 & 0.001 & -15.205 & -22.342 & 0.081 & 0.042 \\
\hline Fourier $\times$ Frank & 0.999 & 9.462 & 0.496 & 0.158 & -14.897 & -22.034 & 0.121 & 0.012 \\
\hline Frank $\times$ AMH & 9.985 & 0.053 & 0.566 & 0.998 & -14.871 & -22.008 & 0.081 & 0.047 \\
\hline Max $\times$ Frank & 2.134 & 9.869 & 0.446 & 0.001 & -14.749 & -21.885 & 0.085 & 0.037 \\
\hline Fourier $\times$ Max & 0.549 & 0.095 & 0.001 & 0.001 & -14.678 & -21.815 & 0.089 & 0.022 \\
\hline $\operatorname{Max} \times$ FGM & 0.098 & -0.316 & 1.000 & 0.999 & -14.677 & -21.814 & 0.090 & 0.017 \\
\hline $\operatorname{Max} \times \mathrm{AMH}$ & 0.098 & -0.316 & 1.000 & 0.999 & -14.677 & -21.814 & 0.090 & 0.017 \\
\hline Max × Min & 0.097 & 1.924 & 0.999 & 0.999 & -14.675 & -21.812 & 0.089 & 0.022 \\
\hline Min $\times$ Frank & 2.135 & 9.835 & 0.453 & 0.001 & -14.674 & -21.811 & 0.087 & 0.032 \\
\hline Independent $\times$ FGM & & 1.000 & 0.001 & 0.001 & -4.200 & -9.553 & 0.223 & 0.001 \\
\hline Independent $\times \mathrm{AMH}$ & & 0.867 & 0.005 & 0.001 & -1.393 & -6.746 & 0.207 & 0.001 \\
\hline $\mathrm{FGM} \times \mathrm{AMH}$ & 0.999 & -0.849 & 1.000 & 0.998 & -0.404 & -7.540 & 0.223 & 0.001 \\
\hline $\operatorname{Min} \times$ FGM & 5.103 & 0.999 & 0.001 & 0.001 & -0.399 & -7.536 & 0.223 & 0.001 \\
\hline Fourier $\times$ FGM & 0.958 & 1.000 & 0.005 & 0.001 & -0.367 & -7.504 & 0.224 & 0.001 \\
\hline Fourier $\times$ AMH & 0.990 & 0.999 & 0.350 & 0.275 & -0.254 & -7.391 & 0.280 & 0.001 \\
\hline $\mathrm{Min} \times \mathrm{AMH}$ & 1.935 & 0.870 & 0.001 & 0.003 & 2.403 & -4.734 & 0.206 & 0.001 \\
\hline Fourier $\times$ Independent & 1.000 & & 0.324 & 0.270 & 6.969 & 1.617 & 0.444 & 0.001 \\
\hline Fourier $\times$ Min & 0.999 & 7.659 & 0.347 & 0.256 & 10.808 & 3.671 & 0.446 & 0.001 \\
\hline Min $\times$ Independent & 8.980 & & 0.067 & 0.001 & 11.353 & 6.000 & 0.462 & 0.001 \\
\hline
\end{tabular}

data.

For the estimation of the hyper-parameters $(\alpha$ and $\beta$ ), one can consider the cross-validation approach instead of the maximum likelihood estimation (MLE) as we did in section 6. After getting the MLE of copula parameters for fixed value of $\alpha$ and $\beta$, one can compare the cross validation copula information criterion (CIC) presented by Jordanger and Tjøstheim (2014). Then choose the parameter estimates that have the minimum of CIC. One may consider a Bayesian approach or expectationmaximization algorithm to estimate the hyper-parameters efficiently.

In our future study, we would extend our copula method to a multivariate case, to develop a generalized composite operator of asymmetric copula family as in Louzada and Ferreira (2016), to apply to the direction data from Kim and Kim (2014), and to incorporate time varying component as in Ara et al. (2017) to our proposed method. R program and datasets are available upon request from the corresponding author. 
Clayton Copula \& Gumbel Copula

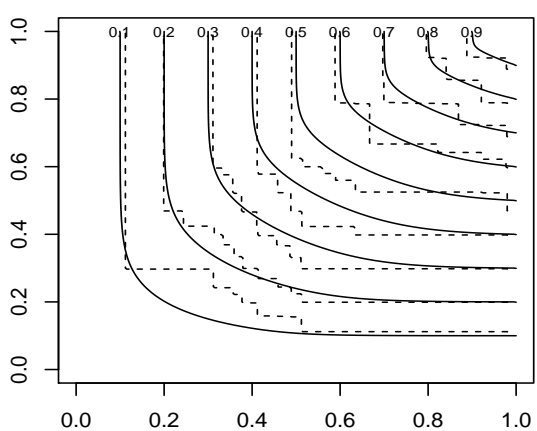

Independent Copula \& Gumbel Copula

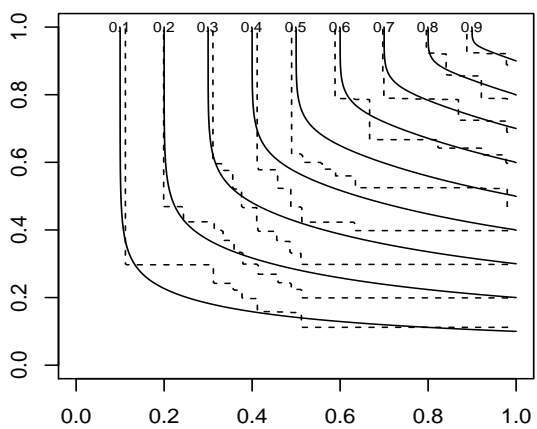

Frank Copula \& Gumbel Copula

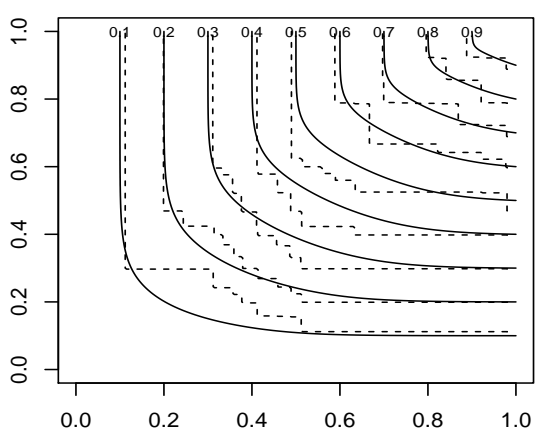

Independent Copula \& Clayton Copula

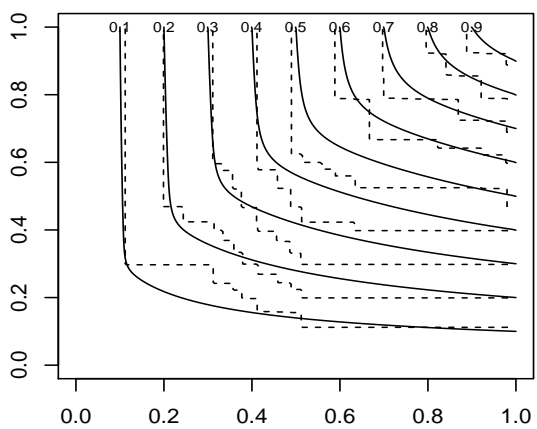

Figure 8: Contour plots of fitted copulas $C_{\theta_{n}}$ (solid line) and empirical copulas $C_{n}$ (dotted line) for the four constructed asymmetric copulas on the economic indicators data.

\section{Acknowledgments}

This work was supported by the National Research Foundation of Korea (NRF) grant funded by the Korea government (MSIP) (No. 2016R1A2B4014518). Lee's work was supported by Basic Science Research Program through the National Research Foundation of Korea (NRF) funded by the Ministry of Education (2017R1A6A3A11032852).

\section{References}

Alfonsi A and Brigo D (2005). New families of copulas based on periodic functions, Journal Communications in Statistics - Theory and Methods, 34, 1437-1447.

Ara A, Louzada F, and Diniz CAR (2017). Statistical monitoring of a web server for error rates: a bivariate time-series copula-based modeling approach, Journal of Applied Statistics, 44, 22872300.

Bouzebda S and Cherfi M (2012). Test of symmetry based on copula function, Journal of Statistical Planning and Inference, 142, 1262-1271. 
Busababodhin P and Amphanthong P (2016). Copula modelling for multivariate statistical process control: a review, Communications for Statistical Applications and Methods, 23, 497-515.

De la Peña VH, Ibragimov R, and Sharakhmetov S (2006). Characterizations of joint distributions, copulas, information, dependence and decoupling, with applications to time series, IMS Lecture Notes-Monograph Series, 2nd Lehmann Symposium-Optimality, 49, 183-209.

Di Bernardino E and Rullière D (2015). On an asymmetric extension of multivariate Archimedean copulas. hal-01147778, from: http://hal.archives-ouvertes.fr/hal-01147778

Durante F (2009). Construction of non-exchangeable bivariate distribution functions, Statist Papers, 50, 383-391.

Genest C, Rémillard B, and Beaudoin D (2009). Goodness-of-fit tests for copulas: a review and a power study, Insurance: Mathematics and Economics, 44, 199-213.

Genest C, Nešlehová J, and Quessy J (2012). Tests of symmetry for bivariate copulas, Annals of the Institute of Statistical Mathematics, 64, 811-834.

Ibragimov R (2009). Copula-based characterizations for higher order Markov processes, Economic Theory, 25, 819-846.

Jordanger LA and Tjøstheim D (2014). Model selection of copulas: AIC versus a cross validation copula information criterion, Statistics \& Probability Letters, 92, 249-255.

Khoudraji A (1995). Constructions a l'etude des couples et a la modelisation de valeurs extremes bivariees (Ph.D. thesis), Université Laval, Quebec, Canada.

Kim D and Kim JM (2014). Analysis of directional dependence using asymmetric copula-based regression models, Journal of Statistical Computation and Simulation, 84, 1990-2010.

Kim JM, Sungur EA, Choi T, and Heo TY (2011). Generalized bivariate copulas and their properties, Model Assisted Statistics and Applications, 6, 127-136.

Kim WH (2014). Dependence structure of Korean financial markets using copula-GARCH model, Communications for Statistical Applications and Methods, 21, 445-459.

Kojadinovic I (2013). An Introduction to the Theory and Practice of Copulas, a PowerPoint File Presented at The Summer School on Copulas for Hydrological Application (STAHY Copula 2013), Hannover, Germany.

Kojadinovic I and Yan J (2010). Modeling multivariate distributions with continuous margins using the copula R package, Journal of Statistical Software, 34, 1-20.

Kojadinovic I, Yan J, and Holmes M (2011). Fast large-sample goodness-of-fit tests for copulas, Statistica Sinica, 21, 841-871.

Liebscher E (2008). Construction of asymmetric multivariate copulas, Journal of Multivariate Analysis, 99, 2234-2250.

Lowin JL (2010). The Fourier copula: theory \& applications, SSRN Electronic Journal, from: http:// ssrn.com/abstract $=1804664$

Louzada F and Ferreira PH (2016). Modified inference function for margins for the bivariate clayton copula-based SUN Tobit Model, Journal of Applied Statistics, 43, 2956-2976

Mesiar R and Najjari V (2014). New families of symmetric/asymmetric copulas, Fuzzy Sets and Systems, 252, 99-110.

Mukherjee S, Jafari F, and Kim JM (2015). Optimization of Spearman's Rho, Revista Colombiana De Estadística, 38, 209-218.

Nayland College (2004). American new cars and truck of 2004, NZ, from: http://maths.nayland.school. nz/Year_13_Maths/3.9_Bivariate_data/exemplars/American_New_Cars_and_Trucks_of_2004.csv

Nelsen RB (2006). An Introduction to Copulas (2nd ed), Springer, New York.

Nelsen RB (2007). Extremes of nonexchangeability, Statistical Papers, 48, 329-336. 
Quessy JF and Bahraoui T (2013). Graphical and formal tools for the symmetry of bivariate copulas, Canadian Journal of Statistics, 41, 637-656.

Quessy JF and Kortbi O (2016). Minimum-distance statistics for the selection of an asymmetric copula in Khoudraji's class of models, Statistica Sinica, 26, 177-204.

Rodríguez-Lallena JA and Úbeda-Flores M (2004). A new class of bivariate copulas, Statistics \& Probability Letters, 66, 315-325.

Sklar A (1959). Fonctions de répartition à n dimensions et leurs marges, Publications de I'Institut Statistique de I'Université de Paris, 8, 229-231.

Schweizer B and Sklar A (1983). Probabilistic Metric Spaces, Elsevier, New York.

Statistics Korea. Korean Statistical Information Service, from: http://kosis.kr/statHtml/statHtml.do? orgId=376\&tblId=DT_376_100_SDMZ021V__1\&vw_cd=MT_ZTITLE\&list_id=M2_A0001_A00 $01 \&$ seqNo $=\&$ lang_mode $=$ ko\&language $=$ kor\&obj_varid $=\& i t m \_i d=\&$ conn $\_$path $=E 1$

Wu S (2014). Construction of asymmetric copulas and its application in two-dimensional reliability modeling, European Journal of Operational Research, 238, 476-485. 